\title{
Social support for healthy eating: development and validation of a questionnaire for the French-Canadian population
}

\author{
Elise Carbonneau ${ }^{1,2, *}$, Maude Bradette-Laplante ${ }^{1,2}$, Benoît Lamarche ${ }^{1,2}$, \\ Véronique Provencher ${ }^{1,2}$, Catherine Bégin ${ }^{1,3}$, Julie Robitaille ${ }^{1,2}$, Sophie Desroches ${ }^{1,2}$, \\ Marie-Claude Vohl ${ }^{1,2}$, Louise Corneau ${ }^{1,2}$ and Simone Lemieux ${ }^{1,2}$ \\ ${ }^{1}$ Institute of Nutrition and Functional Foods, Laval University, 2440 boulevard Hochelaga, Québec, QC, Canada, \\ GIV OA6: ${ }^{2}$ School of Nutrition, Laval University, Québec, QC, Canada: ${ }^{3}$ School of Psychology, Laval University, \\ Québec, QC, Canada
}

Submitted 31 October 2017: Final revision received 27 March 2018: Accepted 5 April 2018; First published online 28 May 2018

\begin{abstract}
Objective: The present study aimed to develop and validate a questionnaire assessing social support for healthy eating in a French-Canadian population.

Design: A twenty-one-item questionnaire was developed. For each item, participants were asked to rate the frequency, in the past month, with which the actions described had been done by family and friends in two different environments: (i) at home and (ii) outside of home. The content was evaluated by an expert panel. A validation study sample was recruited and completed the questionnaire twice. Exploratory factor analysis was performed on items to assess the number of subscales. Internal consistency reliability was assessed using Cronbach's $a$. Test-retest reliability was evaluated with intraclass correlations between scores of the two completions.

Setting: Online survey.

Subjects: Men and women from the Québec City area ( $n$ 150).

Results: The content validity assessment led to a few changes, resulting in a twenty-two-item questionnaire. Exploratory factor analysis revealed a two-factor structure for both environments, resulting in four subscales: supportive actions at home; non-supportive actions at home; supportive actions outside of home; and non-supportive actions outside of home. Two items were removed from the questionnaire due to low loadings. The four subscales were found to be reliable (Cronbach's $a=0 \cdot 82-0 \cdot 94$; test - retest intraclass correlation $=0 \cdot 51-0 \cdot 70$ ).

Conclusions: The Social Support for Healthy Eating Questionnaire was developed for a French-Canadian population and demonstrated good psychometric properties. This questionnaire will be useful to explore the role of social support and its interactions with other factors in predicting eating behaviours.
\end{abstract}

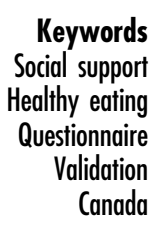

From an ecological perspective, eating behaviours are determined by a wide range of individual, social and environmental factors as well as by their interactions ${ }^{(1)}$. To effectively promote healthy eating, it is critical to better understand how these various factors influence eating behaviours. Individual factors have been extensively studied in the last decades ${ }^{(2,3)}$ and lately an increasing number of studies have documented the association between the physical environment and food choices $^{(4,5)}$. However, the influence of social factors on healthy eating has been less studied.

Social or interpersonal factors are likely to influence food choices and eating behaviours not only since meals are frequently shared with other people, but also due to food-related tasks, such as food planning, procurement and preparation, which are frequently divided among family members, partners or roommates ${ }^{(6)}$. While children's eating behaviours are largely determined by parents' influence ${ }^{(7,8)}$, as a person grows up, a part of the social influence shifts from parents to friends, partner and colleagues ${ }^{(9-11)}$.

Social support can be defined as 'a transactional communicative process, including verbal and/or nonverbal communication, that aims to improve an individual's feelings of coping, competence, belonging, and/or esteem, $^{(12)}$. To adequately assess the influence of social support on healthy eating, it is crucial that a validated tool be used. The most commonly used scales assessing social support from family and friends for diet and exercise 
behaviours have been developed by Sallis et $a l .{ }^{(13)}$ and later modified or adapted by numerous authors for different contexts ${ }^{(14-19)}$. Using the original scales or items derived from it, authors have observed associations between social support from family and friends and measures of healthy eating ${ }^{(13,17,19,20)}$.

To our knowledge, no questionnaire in French has been developed to specifically assess social support in the context of adherence to a healthy diet. It is recognized that tools need to be adapted and validated if they are to be used across countries ${ }^{(21)}$. French-Canadians from the Province of Québec have been found to differ from other Canadians with respect to food intakes and attitudes towards eating ${ }^{(22,23)}$, suggesting that eating-related questionnaires validated in other parts of North America may not be suitable for them. Therefore, the purpose of the present study was to develop and validate a new questionnaire assessing social support for healthy eating in an adult French-Canadian population.

\section{Methods}

\section{Development of the items}

Based on Sallis et $a l^{(13)}$, it was decided that a questionnaire assessing social support from two categories of close others, namely family and friends, would be developed. Participants were asked to rate the frequency, in the past month, with which both family and friends had done the different actions described. It was decided that the actions would relate to the general concept of healthy eating or to junk foods. The majority of the items of the French-Canadian questionnaire were generated based on existing validated tools $\mathrm{s}^{(13,14,24,25)}$. Other items were developed by the research team. A total of twenty-one items were developed. As suggested by Kiernan et al. ${ }^{(25)}$, various forms of social support were assessed through the items, such as verbal support (e.g. '[...] encouraged me to eat healthy foods when I was tempted to eat junk food') and behavioural support (e.g. '[...] ate healthy foods in front of me'). We also decided to add items pertaining to the social environment during meals (e.g. '[...] contributed to create a pleasant atmosphere at mealtime'). According to the recommendations in Kiernan et al. ${ }^{(25)}$, we opted to use a five-point Likert scale ('never', 'rarely', 'sometimes', 'often', 'very often'). It was also decided that a 'not applicable' option would be added to alleviate pressure on participants to answer. Using the cold deck imputation procedure, all 'not applicable' answers were replaced by the 'never' option, based on the questionnaire from Ball and Crawford ${ }^{(14)}$ in which 'not applicable' and 'never' constitute the same answer option.

\section{Participants and procedures}

The questionnaire was developed and validated in the context of a larger study aiming at validating a series of questionnaires on determinants of healthy eating ${ }^{(26-28)}$ and biomarkers of fruit and vegetable intake. The validation process for the Social Support for Healthy Eating Questionnaire was divided into three steps, namely an expert panel evaluation, a pre-test and a validation study.

\section{Expert panel}

Content validity was assessed by an expert panel composed of four nutrition researchers, one registered dietitian and one psychology researcher, all familiar with questionnaire development and validation. Content validity involves an assessment of the items to ensure representation of the construct (i.e. social support for healthy eating) ${ }^{(29)}$. The experts rated each item of the questionnaire on four-point scales for four criteria: relevance ('not relevant' $=1$ to 'very relevant' $=4$ ), clarity ('not clear' $=1$ to 'very clear' $=4$ ), simplicity ('not simple' $=1$ to 'very simple' $=4$ ) and ambiguity ('ambiguous' $=1$ to 'explicit'=4). Evaluations from the experts were combined to yield a content validity index for each item. According to DiIorio ${ }^{(29)}$, a minimum level of $80 \%$ was considered for an adequate content validity. Items with a content validity index lower than the cut-off needed to be modified or removed from the questionnaire.

\section{Pre-test}

According to the questionnaire pre-testing sample size recommendation $^{(30)}$, a convenience sample consisting of thirty-one participants was recruited from an internal list of people willing to participate in clinical studies, to assess the acceptability and comprehension of the items. Participants were men and women from the Québec City metropolitan area and were aged between 18 and 65 years old. Participants had to have at least minimal informatics skills since questionnaires were completed online.

To assess face validity (i.e. whether the items seemed to measure what the developers claim they measure ${ }^{(29)}$ ), participants from the pre-test were asked to complete the questionnaires online and to comment on the ambiguity of the items in a comments box after each item.

\section{Validation study}

A convenience sample of seventy-five women and seventy-five men was used for the validation study. Participants were recruited through electronic mailing lists comprising Laval University students and employees as well as people interested in participating in studies at the Institute of Nutrition and Functional Foods. Inclusion criteria were identical to those used for the pre-test. Participants suffering from conditions affecting intestinal absorption were excluded since blood biomarkers of fruit and vegetable intake were also being validated in the study. Pregnancy and lactation were exclusion criteria. Eligible participants came to the research centre for a blood sample and anthropometric measurements ${ }^{(31)}$. 
Within a month after coming to the laboratory, participants had to complete a series of questionnaires to be validated on the Internet platform of the study. Questionnaires were assigned to participants in a random order. Following a two-week delay, participants were asked to complete each questionnaire a second time within a onemonth period. The completion time for the series of questionnaires was approximately $40 \mathrm{~min}$.

Participants received a financial compensation of \$CAN 50 for their participation in the study. The study was conducted according to the guidelines laid down in the Declaration of Helsinki ${ }^{(32)}$.

\section{Statistical analyses}

The analyses described in this section were performed on data derived from the validation study. An exploratory factor analysis (EFA) was performed on the items to assess the number of factors (or subscales) for the questionnaire, aiming at retaining as few factors as possible while explaining most of the variation in the data. The modified eigenvalue-greater-than-one rule ${ }^{(33)}$ and the scree plot $^{(34)}$ were used to adequately identify the number of factors in the questionnaire. The sample size of the validation study ( $n$ 150) respected the recommended $5: 1$ participant-to-item ratio for factor analyses ${ }^{(35)}$. Internal consistency reliability was assessed using Cronbach's $\alpha$ coefficients with data from the first completion of the questionnaire. The test-retest reliability was assessed with intraclass correlation analyses conducted between scores of the two completions. Statistical tests were two-sided and associations or differences at $P<0.05$ were considered significant. Analyses were performed using the statistical software package SAS version 9.4 (2013).

\section{Results}

\section{Expert panel}

Content validity

Of the twenty-one items evaluated, only one item had a content validity index lower than $80 \%$ and it was removed from the questionnaire. One item was also removed from the questionnaire because it was judged to be too similar to another item. Seven items were slightly modified following comments from the experts. Finally, to address recommendations by the experts, three items were added to the questionnaire. These changes resulted in a twentytwo-item questionnaire (see Table 2).

\section{Pre-test}

Participants in the pre-test were seventeen women and fourteen men (mean age: 45.6 (SD 13.9) years).

\section{Face validity}

Four participants did not complete the social support questionnaire, therefore twenty-seven participants were included for the face validity. Comments expressed by the participants did not lead to any change in the formulation of the twenty-two items. However, following some comments on the classification of close others (e.g. 'Is an adult child no longer living at home considered as a family member or a friend?'), the labels were changed to facilitate understanding. Therefore, 'family' (defined as people living with you) was changed for 'home' (defined as people living with you, e.g. family members, partner, roommate). 'Friends' (defined as friends, colleagues, acquaintances) was replaced by 'outside of home' (defined as people with whom you share meals, but who do not live with you, e.g. friends, colleagues).

\section{Validation study}

Of the 150 participants of the validation study, one participant dropped out of the study before completing the questionnaires and one participant did not complete the social support questionnaire. Characteristics of the 148 remaining participants (mean age: 47.5 (SD 13.4) years) are presented in Table 1 . The mean completion time of the questionnaire was 5.5 (SD 1.9) $\mathrm{min}$.

\section{Exploratory factor analysis}

Since items of the questionnaire were rated separately for two categories (i.e. 'home' and 'outside of home'), EFA was performed separately for each category. The significance of Bartlett's test of sphericity ('home': $\chi^{2}=1923.48, P<0.0001$; 'outside of home': $\chi^{2}=1130.61$, $P<0.0001)$ and the Kaiser-Meyer-Olkin test of sampling adequacy (measures of sample adequacy $>0.50$ ) revealed that our sets of items had adequate common variance,

Table 1 Characteristics of the validation study participants ( $n$ 148), Québec City area, Canada

\begin{tabular}{lrr}
\hline & $n$ & $\%$ \\
\hline Female & 73 & $49 \cdot 3$ \\
Ethnicity & & \\
$\quad$ Caucasian & 141 & $95 \cdot 3$ \\
Highest level of education & & \\
$\quad$ High school & 14 & $9 \cdot 5$ \\
$\quad$ College & 45 & $30 \cdot 4$ \\
$\quad$ University & 89 & $60 \cdot 1$ \\
Occupation & & \\
$\quad$ Worker & 101 & $68 \cdot 2$ \\
Retired & 32 & $21 \cdot 6$ \\
Student & 9 & $6 \cdot 1$ \\
No job & 3 & $2 \cdot 0$ \\
$\quad$ Prefer not to answer & 3 & $2 \cdot 0$ \\
Living with & & \\
$\quad$ Partner only & 56 & $37 \cdot 8$ \\
Partner and children & 30 & $20 \cdot 3$ \\
Children only & 5 & $3 \cdot 4$ \\
Family member (other than partner and children) & 6 & $4 \cdot 0$ \\
$\quad$ Roommate & 5 & $3 \cdot 4$ \\
$\quad$ Alone & 46 & $31 \cdot 1$ \\
\hline
\end{tabular}


Table 2 Questionnaire items, with their factor loadings, for assessing social support for healthy eating in a French-Canadian population

\begin{tabular}{|c|c|c|}
\hline & Factor $1^{*}$ & Factor $2^{*}$ \\
\hline 1. [...] proposed that we eat healthier & $0.66 / 0 \cdot 40$ & \\
\hline 2. [...] minimized the importance of consuming healthy foods & & $0.59 / 0.66$ \\
\hline 3. [...] made positive comments on my consumption of healthy foods & $0.70 / 0.59$ & \\
\hline 4. [...] ate healthy foods in front of me & $0.83 / 0.67$ & \\
\hline 5. [...] made negative comments when I was eating less healthily & $0.47 / 0.45$ & \\
\hline 6. [...] encouraged me to eat healthy foods when I was tempted to eat junk foods & $0.55 / 0.30$ & \\
\hline 7. [...] ate junk foods in front of me & & $0.35 / 0 \cdot 49$ \\
\hline 8. [...] made positive comments on healthy foods' taste & $0.84 / 0 \cdot 79$ & \\
\hline 9. [...] preferred to eat alone than with me† & - & - \\
\hline 10. [...] hampered my efforts to eat more healthily & & $0.67 / 0.72$ \\
\hline 11. [...] encouraged me to buy healthy foods & $0 \cdot 86 / 0 \cdot 73$ & \\
\hline 12. $[\ldots]$ gave me ideas to eat more healthy foods & $0.78 / 0.67$ & \\
\hline 13. [...] said that healthy foods do not taste good & & $0.76 / 0.59$ \\
\hline 14. [...] contributed to create a pleasant atmosphere at mealtime & $0.82 / 0.52$ & \\
\hline 15. [...] insisted for me to eat junk foods & & $0.68 / 0 \cdot 66$ \\
\hline 16. [...] took their meals with me & $0.80 / 0.46$ & \\
\hline 17. [...] made negative comments on my healthy foods consumption & & $0.68 / 0.74$ \\
\hline 18. [...] praised the advantages of eating healthy foods & $0.87 / 0 \cdot 81$ & \\
\hline 19. [...] criticized the healthy foods that I served them & & $0.73 / 0.63$ \\
\hline 20. [...] contributed to create a tense atmosphere at mealtime & & $0.65 / 0.33$ \\
\hline 21. [...] proposed that we eat in front of the televisiont & - & - \\
\hline 22. [...] listened to my opinions regarding healthy eating even when they disagree & $0.71 / 0 \cdot 48$ & \\
\hline
\end{tabular}

The French version of the instrument was validated. All items were translated from French to English using the back-translation procedure and should not be used in their English version without validation.

*Factor loadings are presented as: 'home'/'outside of home'. Close others at 'home' are defined as people living with you (e.g. family members, partner, roommate). Close others 'outside of home' are defined as people with whom you share meals, but who do not live with you (e.g. friends, colleagues.) †ltems that were removed after the exploratory factor analysis.

justifying the use of an $\mathrm{EFA}^{(36)}$. When looking at data from 'home' and 'outside of home', respectively five and four factors had eigenvalues (including their $95 \% \mathrm{CI}$ ) greater than $1 \cdot 0$. However, the analysis of the scree plots from the EFA revealed a notable difference in the slope after the first two factors for 'home' and 'outside of home'. Therefore, it was decided that the items of the questionnaire would be divided into two factors for both 'home' and 'outside of home'. The two factors accounted for $82.88 \%$ of the variance in the data from 'home' and for $81.17 \%$ of the variance in the data from 'outside of home'. To obtain simple and interpretable factors, and since factors were expected to covary, oblique promax rotations were used for both the 'home' and 'outside of home' categories. Using a minimum loading cut-off of 0.30 or higher ${ }^{(37)}$, two items did not load on any factor and were therefore removed from the questionnaire (see Table 2).

With this factor structure, twelve items were found to load on the first factor and eight items on the second factor, as seen in Table 2. The same factor structure was obtained for both 'home' and 'outside of home'. The factors were named according to what the items of each subscale have in common. Therefore, the first factor was named 'supportive actions' (e.g. '[...] proposed that we eat healthier') and the second was named 'non-supportive actions' (e.g. '[...] criticized the healthy foods I prepared'). Since the questionnaire is divided into two factors and two categories (i.e. 'home' and 'outside of home'), it was decided that the questionnaire would be scored on four distinct subscales: supportive actions at home; nonsupportive actions at home; supportive actions outside of home; and non-supportive outside of home. Subscale scores were obtained by calculating the mean of the items. Thus, higher scores for the supportive and the nonsupportive scales mean a higher frequency of these types of action. To verify if the presence of supportive actions reflects the absence of non-supportive actions, correlations between the two factors were assessed. Supportive actions and non-supportive actions score were significantly correlated within the 'home' category $(r=0.27$, $P=0.0010)$ and the 'outside of home' category $(r=0.34$, $P<0 \cdot 0001)$. Mean scores for the four subscales are presented in Table 3 .

\section{Internal consistency reliability}

As seen in Table 3, all scores were considered internally reliable, with all Cronbach's $\alpha$ coefficients being $>0 \cdot 70$.

\section{Test-retest reliability}

Intraclass correlation coefficients between both completions were calculated for the participants who completed the scale twice ( $n$ 146). As shown in Table 3, correlations indicate moderate reliability ${ }^{(38)}$ for all subscales $(\rho=0.51$ to $0 \cdot 67)$. The mean lapse between test and retest was 40.9 (sD 11.5) d.

\section{Discussion}

The purpose of the present study was to develop and validate a questionnaire assessing social support for healthy eating for a French-Canadian population. We 
Table 3 Scores obtained by participants on the four subscales, Cronbach's a coefficients and intraclass correlation coefficients (ICC) in the validation of the questionnaire for assessing social support for healthy eating in a French-Canadian population

\begin{tabular}{lccccc}
\hline Subscale & Mean score† & SD & Cronbach's ał & ICC§ & Cl \\
\hline Supportive actions at home & 2.83 & 1.11 & 0.94 & 0.65 & $0.55,0.73$ \\
Non-supportive actions at home & 1.41 & 0.48 & 0.85 & 0.67 & $0.57,0.75$ \\
Supportive actions outside of home & 2.50 & 0.75 & 0.86 & 0.51 & $0.38,0.62$ \\
Non-supportive actions outside of home & 1.57 & 0.51 & 0.82 & 0.65 & $0.55,0.73$ \\
\hline
\end{tabular}

Scores are on a maximum of 5 points. Higher scores for the supportive and the non-supportive scales mean a higher frequency of these types of action.

†n 148.

$\ddagger n 142$

§n 146.

decided to create a full questionnaire and proceeded to a complete validation of the items developed, unlike many authors who have assessed social support using only a few items from other validated questionnaires without proceeding to a validation of these items taken separately. We decided to create a questionnaire targeting the whole population, and not only people wishing to adopt a healthier lifestyle. Therefore, items pertain to the global concepts of healthy eating and junk foods. Other questionnaires already exist to assess various forms of goal support that can be provided by family and friends to an individual aiming at changing a behaviour (e.g. Koestner et $\left.a l .{ }^{(39)}\right)$. Items were generated based on existing questionnaires or developed by the research team. Given that the items developed by Sallis et al. ${ }^{(13)}$ are largely used in the literature, this questionnaire was an important source of inspiration for the development of our questionnaire. We were also inspired by the modifications proposed by Ball and Crawford ${ }^{(14)}$ and Kiernan et al. ${ }^{(25)}$, such as using the same items for both types of close others.

Face validity was used to assess pre-test participants' understanding of the items. This led to an important improvement in the wording used to describe the two categories of close others, to reduce ambiguity (change from 'family' to 'home' and from 'friends' to 'outside of home'). This change in the labels led to a classification focusing on the context of the interactions more than on the nature of the relationship itself. We think that our classification will bring interesting information about the role of social support from important others whether living or not with them, which is not the case with other existing social support questionnaires. When living with someone, whether it is a partner, a family member or a friend, numerous interactions around food and eating are likely to happen, while preparing and sharing meals. However, our results may not be directly comparable to most other social support questionnaires using 'family/friends' categories.

Items were developed to assess three types of social support, namely verbal support, behavioural support and social environment during meals. Therefore, it was expected that the EFA would yield to a three-factor structure. However, the two-factor structure uncovered by the analysis is in line with the structure of the original social support scales by Sallis et $a l^{(13)}$ and the adapted versions $^{(14,25)}$. The EFA also resulted in the removal of two items pertaining to the meal environment. Those items may not be directly related to the social support concept.

The four subscales were found to be internally reliable, meaning that items within each subscale related well with one another. The two factors were moderately correlated within the 'home' category and the 'outside of home' category. These results indicate that the factors are tapping distinct concepts and that the use of two subscales is relevant. Sallis et $a l^{(13)}$ reported stronger correlations between the different forms of support (family: $r=0.45$; friends: $r=0.38$ ), but yet considered that the factors did not overlap. The correlations we observed between the two types of actions suggest that many participants received both supportive and non-supportive actions from their close others. In future research, it would be interesting to assess if the presence of non-supportive actions alleviates the influence of supportive actions on healthy eating behaviours. In the present study, participants were not asked to specify who did which action. Therefore, we do not know if some individuals are perceived to be both supportive and non-supportive, or if the various actions reported by participants originate from different persons.

In the present study, intraclass correlation coefficients indicated moderate test-retest reliability (i.e. coefficients between 0.51 and 0.67 ) for all four subscales. The delay between the two completions was variable, from 14 to $99 \mathrm{~d}$. Few authors have assessed this type of validation of a social support for healthy eating questionnaire. Sallis et al. ${ }^{(13)}$ obtained stronger correlation coefficients, varying from 0.57 to 0.86 , but the time period between the two completions is not mentioned in the article. Baranowski et $a l^{(24)}$ assessed a six-week test-retest reliability of their questionnaire on family social support for purchasing fruits and vegetables, obtaining correlation coefficients that were also higher than in the present study $(r=0.74$ and 0.73 ). Our lower test-retest coefficient can be partly explained by the variable time period between the two completions due to the study design. It is also important to note that the intraclass correlation coefficients were higher for 'supportive actions at home' than for 'supportive actions outside of home' subscales $(\rho=0.65 v . \rho=0.51)$. This suggests that social support outside of home, from friends, colleagues and acquaintances, is more likely to vary with time. Accordingly, it is possible that there is more stability in people with whom one shares meals at 
home than outside of home. The varying nature of social support stresses the importance of choosing wisely the time frame of the questionnaire. Therefore, when social support is compared with food intake, it is all the more important that the time period of the social support questionnaire matches with the period covered by the food intake questionnaire.

\section{Strengths and limitations}

Some limitations of the present study should be acknowledged. The study population was mostly Caucasian and highly educated, and therefore not representative of the whole French-Canadian population ${ }^{(40)}$, which can limit the external validity. Also, the current validation study could have benefited from a comparison between the newly developed questionnaire and other tools assessing social support, as it would have increased the content validity. However, other authors have compared social support for healthy eating with a general social support score and have found no significant association, suggesting that specific types of support may vary in quantity and may come from different people than what general social support scales usually measure ${ }^{(13)}$.

An important strength of the study is the fact that it was designed expressly for the validation of questionnaires. The three main steps, namely the expert panel, the pre-test and the validation study, were followed for each questionnaire to be validated, resulting in a rigorous validation process.

\section{Conclusions}

The present study aimed at validating the Social Support for Healthy Eating Questionnaire, developed in a FrenchCanadian population. The design of the questionnaire was inspired from existing tools and demonstrated good psychometric properties. Data from test-retest analyses suggest that social support may vary within relatively short periods of time. Hence, research schedules should be carefully planned to ensure that social support is assessed as closely as possible to other measures related to food intake and behaviours. This questionnaire will be useful to explore the role of social support and its interactions with other factors in predicting healthy dietary habits and eating behaviours. The questionnaire developed in the present study is a valid and reliable tool for the French-Canadian adult population, but extra validation is recommended if the tool is to be used in other populations.

\section{Acknowledgements}

Acknowledgements: The authors express their gratitude to the participants for their involvement in the study. The authors would like to acknowledge the work of Catherine Laramée who contributed significantly to the conduction of the study. Financial support: The present study was supported by the Canadian Institutes of Health Research (CIHR; grant number FHG 129921). E.C. is a recipient of a scholarship from the Food Quality Observatory. The funders had no role in the design, analysis or writing of this article. Conflict of interest: None. Authorship: S.L., B.L., V.P., C.B., J.R., S.D. and M.-C.V. contributed in designing the study. L.C. and M.B.-L. were responsible for carrying out the study. M.B.-L., S.L. and E.C. were responsible for developing the questionnaire. V.P., C.B., J.R., S.D., M.C.-V. and L.C. were members of the expert panel. E.C. analysed the data and wrote the article. All co-authors revised and approved the final version of the paper. Ethics of buman subject participation: This study was conducted according to the guidelines laid down in the Declaration of Helsinki and all procedures involving human subjects were approved by the Research Ethics Committee at Laval University. Implicit informed consent was obtained from the pre-test participants and all participants from the validation study gave written informed consent.

\section{References}

1. McLeroy KR, Bibeau D, Steckler A et al. (1988) An ecological perspective on health promotion programs. Health Educ Q 15, 351-377.

2. Guillaumie L, Godin G \& Vezina-Im LA (2010) Psychosocial determinants of fruit and vegetable intake in adult population: a systematic review. Int J Behav Nutr Phys Act 7, 12.

3. Shaikh AR, Yaroch AL, Nebeling L et al. (2008) Psychosocial predictors of fruit and vegetable consumption in adults: a review of the literature. Am J Prev Med 34, 535-543.

4. Giskes K, van Lenthe F, Avendano-Pabon M et al. (2011) A systematic review of environmental factors and obesogenic dietary intakes among adults: are we getting closer to understanding obesogenic environments? Obes Rev 12, e95-e106.

5. Kamphuis CB, Giskes K, de Bruijn GJ et al. (2006) Environmental determinants of fruit and vegetable consumption among adults: a systematic review. Br J Nutr 96, 620-635.

6. Bove CF \& Sobal J (2006) Foodwork in newly married couples. Food Cult Soc $\mathbf{9}, 69-89$.

7. Birch LL \& Fisher JO (1998) Development of eating behaviors among children and adolescents. Pediatrics 101, 539-549.

8. Faith MS, Scanlon KS, Birch LL et al. (2004) Parent-child feeding strategies and their relationships to child eating and weight status. Obes Res 12, 1711-1722.

9. Ng JYY, Ntoumanis N, Thøgersen-Ntoumani C et al. (2013) Predicting psychological needs and well-being of individuals engaging in weight management: the role of important others. Appl Psychol Health Well Being 5, 291-310.

10. Kalavana TV, Maes S \& De Gucht V (2010) Interpersonal and self-regulation determinants of healthy and unhealthy eating behavior in adolescents. J Health Psychol 15, 44-52.

11. Marcoux BC, Trenkner LL \& Rosenstock IM (1990) Social networks and social support in weight loss. Patient Educ Couns 15, 229-238.

12. Marifran M \& Gibb HJ (2011) Linking health communication with social support. In Health as Communication Nexus: $A$ 
Service-Learning Approach, p. 184 [M Mattson and J Gibb Hall, editors]. Dubuque, IA: Kendall Hunt Publishing Company.

13. Sallis JF, Grossman RM, Pinski RB et al. (1987) The development of scales to measure social support for diet and exercise behaviors. Prev Med 16, 825-836.

14. Ball K \& Crawford D (2006) An investigation of psychological, social and environmental correlates of obesity and weight gain in young women. Int J Obes (Lond) $\mathbf{3 0}$, 1240-1249.

15. William JE, Agate ST, Cason KL et al. (2010) Predictors of social support for healthy nutrition behaviors among African American adolescents. https://projects.ncsu.edu/ffci/ publications/2010/v15-n1-2010-spring/williams-agate-casongriffin.php (accessed April 2018).

16. Swan E, Bouwman L, Hiddink GJ et al. (2015) Profiling healthy eaters. Determining factors that predict healthy eating practices among Dutch adults. Appetite 89, 122-1230.

17. Williams LK, Thornton L \& Crawford D (2012) Optimising women's diets. An examination of factors that promote healthy eating and reduce the likelihood of unhealthy eating. Appetite 59, 41-46.

18. Yates BC, Pullen CH, Santo JB et al. (2012) The influence of cognitive-perceptual variables on patterns of change over time in rural midlife and older women's healthy eating. Soc Sci Med 75, 659-667.

19. Ball K, Crawford D \& Mishra G (2006) Socio-economic inequalities in women's fruit and vegetable intakes: a multilevel study of individual, social and environmental mediators. Public Health Nutr 9, 623-630.

20. Evans GL, McNeil LH, Laufman L et al. (2009) Determinants of low-fat eating behaviors among midlife African American women. J Nutr Educ Behav 41, 327-333.

21. Beaton DE, Bombardier C, Guillemin F et al. (2000) Guidelines for the process of cross-cultural adaptation of self-report measures. Spine (Phila Pa 1976) 25, 3186-3191.

22. Garriguet D (2004) Nutrition: Findings from the Canadian Community Health Survey: Overview of Canadians' Eating Habits. Ottawa: Statistics Canada.

23. Canadian Foundation for Dietetic Research (2013) Tracking Nutrition Trends 2013. Ontario: CFDR.

24. Baranowski T, Cullen KW \& Baranowski J (1999) Psychosocial correlates of dietary intake: advancing dietary intervention. Annu Rev Nutr 19, 17-40.

25. Kiernan M, Moore SD, Schoffman DE et al. (2012) Social support for healthy behaviors: scale psychometrics and prediction of weight loss among women in a behavioral program. Obesity (Silver Spring) 20, 756-764.
26. Carbonneau E, Carbonneau N, Lamarche B et al. (2016) Validation of a French-Canadian adaptation of the Intuitive Eating Scale-2 for the adult population. Appetite 105, 37-45.

27. Carbonneau E, Robitaille J, Lamarche B et al. (2017) Development and validation of the Perceived Food Environment Questionnaire in a French-Canadian population. Public Health Nutr 20, 1914-1920.

28. Bradette-Laplante M, Carbonneau E, Provencher V et al. (2017) Development and validation of a nutrition knowledge questionnaire for a Canadian population. Public Health Nutr 20, 1184-1192.

29. DiIorio CK (2006) Measurement in Health Behavior: Methods for Research and Evaluation. San Francisco, CA: Jossey-Bass.

30. Perneger TV, Courvoisier DS, Hudelson PM et al. (2015) Sample size for pre-tests of questionnaires. Qual Life Res $\mathbf{2 4}$, 147-151.

31. Callaway C, Chumlea W \& Bouchard C (1988) Standardization of anthropometric measurements. In The Airlie (VA) Consensus Conference, pp. 39-80 [T Lohman, A Roche \& $\mathrm{R}$ Martorel, editors]. Champaign, IL: Human Kinetics Publishers.

32. World Medical Association (2013) World Medical Association Declaration of Helsinki: ethical principles for medical research involving human subjects. JAMA 310, 2191-2194.

33. Larsen R \& Warne RT (2010) Estimating confidence intervals for eigenvalues in exploratory factor analysis. Behav Res Methods 42, 871-876.

34. Cattell RB (1966) The scree test for the number of factors. Multivariate Behav Res 1, 245-276.

35. Hatcher L (1994) A Step-by-Step Approach to Using the SAS System for Factor Analysis and Structural Equation Modeling. Cary, NC: SAS Institute.

36. Tabachnick BG \& Fidell LS (2007) Using Multivariate Statistics, 5th ed. Boston, MA: Pearson Education.

37. Costello AB \& Osbourne J (2005) Best practices in exploratory factor analysis: four recommendations for getting the most from your analysis. Pract Assess Res Eval 10, issue 7, available at http://pareonline.net/pdf/v10n7.pdf

38. Koo TK \& Li MY (2016) A guideline of selecting and reporting intraclass correlation coefficients for reliability research. J Chiropr Med 15, 155-163.

39. Koestner R, Powers TA, Carbonneau N et al. (2012) Distinguishing autonomous and directive forms of goal support: their effects on goal progress, relationship quality, and subjective well-being. Pers Soc Psychol Bull 38, 1609-1620.

40. Gouvernement du Québec (2016) Québec Handy Numbers, 2016 ed. Québec: Institut de la statistique du Québec. 